\title{
¿Es necesario exigir investigación en el Residentado Médico?
}

\author{
It is obligatory to do research during medical residency?
}

\author{
Theresa J. Ochoa-Woodell ${ }^{1}$
}

\section{Introducción}

No hay duda que la investigación es fundamental para el desarrollo de la medicina, no solo para ampliar el conocimiento sino también y, sobre todo, para mejorar la salud de las personas y de la sociedad en su conjunto. Se necesita de investigación para conocer la epidemiología de las enfermedades y los factores de riesgo, tener mejores métodos diagnósticos, mejorar la efectividad de los tratamientos y para innovar terapias y estrategias de prevención y control. Esta investigación mayoritariamente se realiza en instituciones académicas tales como las universidades e institutos de investigación. En las universidades, la investigación es llevada a cabo por los profesores e investigadores y forma parte del currículo y las competencias del alumno de medicina; $y$, es una pieza fundamental y crítica en los programas de maestría y doctorado en medicina. Sin embargo, es controversial si se debe exigir investigación en los programas de segunda especialidad profesional en medicina, es decir en el residentado médico.

En marzo del 2017 se publicó en las Normas Legales de El Peruano el Decreto Supremo que aprueba el Reglamento de la Ley N 30453 del Sistema Nacional de Residentado Médico (SINAREME). En el artículo 46 sobre el proyecto de investigación se establece:

"Los médicos residentes de especialidad, presentarán al inicio del primer semestre del segundo año, un proyecto de investigación de la especialidad, el que deberá ser evaluado y aprobado por la instancia correspondiente de la institución formadora universitaria de ser el caso, durante el segundo semestre del segundo año", (1).

Por lo expuesto, en nuestro país actualmente es obligatorio hacer un proyecto de investigación durante la residencia. Esta normativa ha ido cambiando en el tiempo, modificándose los requerimientos. Anteriormente, el reglamento exigía a los residentes presentar un trabajo de investigación, es decir, escribir un proyecto de investigación, llevarlo a cabo y presentar los resultados para su aprobación, y así poder titularse como especialista, una vez cumplido con las exigencias del programa.

En este péndulo de normas y exigencias, vale la pena primero analizar cuáles son las ventajas de hacer investigación en el residentado, cuáles son los retos y dificultades y, finalmente, cuáles son las oportunidades y opciones de investigación médica para nuestros residentes (Tabla 1), para luego poder hacer algunas recomendaciones.

En este artículo no se discutirá la situación de la investigación en los programas de maestría y doctorado en medicina en nuestro país, donde la investigación científica es necesaria dado que es una de las exigencias de un grado académico, como en todo el mundo. El principal problema de estos programas a nivel nacional es la baja eficiencia terminal (proporción de alumnos que termina la tesis). Los factores que contribuyen a este problema, en parte son similares a los retos y dificultades que se discutirán más abajo para los residentes.

\section{Ventajas de hacer investigación en el residentado}

Múltiples son los estudios que han descrito las ventajas de promover la investigación en los residentes. Los programas de residentado buscan la formación de médicos especialistas altamente capacitados en su campo clínico y capaces de plantear soluciones a los problemas de salud de su país. Para esto se necesita de habilidades críticas, indagatorias y positivas que se adquieren a través de la investigación. Otra ventaja es la habilidad de enriquecer su quehacer médico con la práctica de la medicina basada en evidencia y el desarrollo de un pensamiento crítico que contribuya a un aprendizaje permanente e independiente.

Cuando se realiza investigación se requiere tener un conocimiento profundo y detallado sobre un tema, lo que permite tener una perspectiva mayor sobre los avances, retos y brechas en la especialidad. Los beneficios del desarrollo de la investigación en el residentado han sido descritos ${ }^{(2)}$.

Se necesita empezar el entrenamiento en investigación en etapas tempranas para asegurar la excelencia de la educación médica y la renovación profesional ${ }^{(3)}$. Se requiere de médicos especialistas capacitados en investigación, quienes serán los próximos profesores universitarios, encargados de la formación de los futuros estudiantes de medicina y de los especialistas. Un estudio en un programa de residencia norteamericano de cirugía ortopédica que tiene dos vías, una tradicional de cinco años y otra de investigación que tiene un año adicional, mostró que los residentes de la vía de investigación tenían, significativamente, más publicaciones en

${ }^{\prime}$ Médica infectóloga. Instituto de Medicina Tropical Alexander von Humboldt. Facultad de Medicina Alberto Hurtado, Universidad Peruana Cayetano Heredia, Lima. 
revistas con revisión por pares que el de la vía tradicional $(71 \%$ vs. $41 \%$, respectivamente), durante la residencia; y, también tenían mayor probabilidad de publicar en dicho tipo de revistas durante su carrera profesional (75\% vs 55\%, respectivamente) (4). Este estudio sugiere que los residentes involucrados en investigación durante su formación participarán con mayor probabilidad en actividad académica durante su vida profesional.

Si bien mucha de la investigación en medicina puede ser hecha por no-médicos, como los investigadores con doctorado $(\mathrm{PhD})$, es cierto que las preguntas relevantes de la investigación biomédica son planteadas por los médicos que conocen las brechas del conocimiento en el campo de su especialidad y saben cómo traducir los hallazgos de la investigación en la práctica clínica cotidiana para beneficio del paciente ${ }^{(5)}$. Entonces, ¿quién mejor que los médicos especialistas para liderar la investigación aplicada en nuestro país?

\section{Retos y dificultades de hacer investigación en el residentado}

La principal dificultad para hacer investigación en el residentado es la falta de tiempo, dado que la carga asistencial en el hospital es fuerte y demandante. Múltiples estudios han documentado esta limitante. Por otro lado, algunos residentes tienen mayor interés que otros en el mundo académico, mostrando diferentes niveles de compromiso y productividad, basado en sus metas profesionales personales.

Justamente, a nivel nacional, uno de los principales retos es la heterogeneidad de los médicos que ingresan a los programas del residentado. Muchos de los residentes no tienen experiencia previa en investigación, dado que hasta hace poco no era obligatorio hacer una tesis o trabajo de investigación para graduarse de la carrera de medicina. Por lo tanto, estos residentes no necesariamente tienen la formación básica en metodología de la investigación, bioestadística, lectura crítica, redacción científica, bioética, etc. Un estudio realizado en residentes graduados de la Universidad Peruana Cayetano Heredia, entre los años 2010 y el 2012, refuerza este concepto, ya que únicamente un tercio de los residentes culminó su proyecto de investigación en el tiempo indicado; y, los principales factores asociados a la culminación del proyecto fueron el haber realizado una tesis en el pregrado y tener un asesor con al menos dos publicaciones científicas por año ${ }^{(6)}$. Aún más crítico, se encontró que un tercio de los residentes fue asesorado por docentes sin ninguna publicación en los últimos cinco años.

Esto nos lleva a plantear la otra gran limitante que es la falta de asesores o mentores con las calificaciones necesarias para tal rol y, además, igual de importante, con el tiempo necesario para dedicarle al residente. Debido a que muchos médicos especialistas no necesariamente tienen experiencia en investigación, sobre todo en los aspectos metodológicos y de estadística, es importante que el médico residente (y lo mismo ocurre para la maestría en medicina) tenga dos asesores: uno médico, que es el experto en el tema de interés de la especialidad, quien va a dar las pautas de lo relevante, de lo innovador y de lo factible del proyecto, y un asesor metodológico que ayudará con la selección del mejor diseño para responder la pregunta de investigación, en el cálculo del tamaño muestral y en el análisis estadístico, que son las principales dificultades metodológicas en un proyecto. Por lo tanto, al no haber muchos asesores metodológicos en las universidades, esta responsabilidad recae en un grupo reducido, muchas veces sobrecargado de trabajo y, por ende, sin tiempo para dedicar al alumno; o, de lo contrario, se obvia de este asesor. Todo esto lleva a la elaboración de proyectos con falencias metodológicas importantes.

Un estudio realizado en Chile mostró que existe una gran intención de desarrollar competencias de investigación clínica en los programas de especialidad médica; pero, se evidenció que existe discrepancia entre la intención de promover la investigación en el residentado y la productividad científica de los docentes y residentes de diferentes especialidades y universidades. Se resaltó que de existir la intención y la necesidad de aumentar la investigación en los residentes se deben dar las condiciones básicas para tal fin ${ }^{(7)}$.

Por otro lado, hay quienes argumentan que la investigación clínica no es fundamental en la residencia. Así, se alude que los residentes son entrenados principalmente para hacer práctica clínica médica en un mundo cada vez más complejo y especializado, y que por lo tanto no deberían perder el tiempo haciendo investigación, y que esta, en la mayoría de las veces se traduce en publicaciones de bajo nivel, con muchos sesgos y que no contribuye realmente a la literatura médica. Además, se argumenta que para lograr investigación científicamente meritoria se requiere de tiempo y dedicación, que no es el caso de los residentes; por lo tanto, las contribuciones científicas deberían dejarse a los investigadores clínicos y a los científicos ${ }^{(8)}$. Sin embargo, uno puede responder que se requiere de la formación de estos futuros investigadores clínicos y científicos.

Otro reto es la falta de ética e integridad científica que, lamentablemente, cada vez está más arraigada en nuestra sociedad. Uno podría argumentar que algunos de estos "errores" son involuntarios, por falta de conocimiento o capacitación. El ejemplo más claro es la copia de ciertas oraciones o párrafos textualmente, con el concepto erróneo que se puede hacer esto si se pone al costado la referencia bibliográfica, cuando en realidad solo está permitido si se pone el texto copiado entre comillas. Sin embargo, fuera de estos errores comunes, quizá no malintencionados, hay evidencia de plagio en el mundo universitario. En un estudio pequeño en 33 tesis de bachiller de medicina, de una universidad pública peruana, se encontró que un tercio de todos los casi 480 párrafos analizados tenían algún grado de plagio $^{(9)}$. Más alarmante aún es el reporte de compra y venta de tesis on-line en el mercado peruano, lo que es una falta gravísima de integridad científica. Dado los altos costos de estos "servicios", los autores del reporte especulan que muy probablemente este mercado sea mayoritariamente usado por alumnos de posgrado 
(residencia, maestría y doctorado) $^{(10)}$. Un análisis longitudinal de casos de plagio y engaño en la maestría de investigación epidemiológica de la UPCH, entre los años 2011-2014, encontró que los alumnos involucrados tenían serias deficiencias incluyendo alta tolerancia al plagio, bajo rendimiento académico y deficiencias en la redacción, con escritura en parches y el tradicional "copiado y pegado"(11). Lo interesante de este estudio es que los directores del programa implementaron medidas correctivas para tratar el problema incluyendo educación en integridad científica y redacción, códigos de honor, búsqueda activa de plagio en los trabajos y una política de "tolerancia cero" a la mala conducta científica. Estas medidas deberían implementarse en todos los programas de segunda especialización de medicina, si se pretende promover investigación en el residentado.

\section{Oportunidades y opciones de} investigación en el residentado

Históricamente, la investigación en medicina ha estado centrada en la investigación básica o clínica. Hoy en día, hay varias opciones en el trabajo académico, más allá de la investigación clínica original como tal, incluyendo investigación en temas de educación médica, seguridad del paciente, mejoramiento de la calidad y administración en salud ${ }^{(3,5)}$. Estos proyectos son importantes en generar soluciones creativas a los problemas del sistema de salud, buscando optimizar la forma de hacer medicina. Una opción usada por muchos programas de residencia es la obligación de presentar resúmenes a los congresos científicos de la especialidad. Estos resúmenes pueden ser de los temas arriba mencionado, así como proyectos pequeños o series de casos. Esto es útil, dado que en su carrera profesional como médicos especialistas asistirán a congresos y presentarán resúmenes; por tanto, durante la residencia se podrían capacitar en esta forma básica de difusión del conocimiento clínico. Los residentes deberían buscar que sus metas académicas estén alineadas con sus intereses profesionales; entonces, deberían hacer un trabajo de investigación o trabajo académico en busca de estas metas, más que cumplir meramente con un requisito.

Como otro ejemplo de tipo de trabajo académico, se describe la importancia y utilidad de enseñar cursos de revisión sistemática y meta-análisis a los residentes. En un programa de residencia de psiquiatría, estos cursos permitieron a sus residentes mejorar sus habilidades en investigación y su productividad académica. Los autores plantearon que esta es una forma factible de enseñar habilidades de investigación que pueden durar de por vida ${ }^{(12)}$.

En la misma línea, hay quienes argumentan que la investigación clínica no es la única forma de hacer actividad académica en la residencia ${ }^{(5,8)}$. Probablemente el trabajo académico más apropiado para los futuros médicos especialistas podría ser presentación de casos clínicos, lectura crítica de la literatura, cartas al editor criticando artículos científicos, entre otros ${ }^{(5)}$. El objetivo de esta actividad académica es que los residentes puedan entender las contribuciones científicas en la medicina. Así mismo, algunas revistas médicas del extranjero, como el CJEM (Canadian Journal of Emergency Medicine) están interesadas en publicar el trabajo de los residentes, incluyendo correspondencia clínica (por ejemplo, reporte de casos e imágenes), comentarios, reportes cortos de educación médica, así como artículos originales breves $^{(3)}$. A nivel nacional, habría que proponer a las revistas indizadas en PubMed, que puedan generar espacios dedicados a los residentes como lo estable el CJEM. Esta revista está interesada en publicar artículos de los residentes dado que reconocen la importancia de iniciar el entrenamiento en investigación tempranamente, para sentar las bases de los futuros académicos y líderes de la medicina ${ }^{(3)}$.

Por otro lado, hay otros que argumentan que la investigación no debería ser obligatoria para todos los residentes ${ }^{(5,8)}$. Si bien hay muchas razones por la que la mayoría de los residentes deberían hacer investigación, como se ha discutido en la primera parte de este artículo, no todos tienen el interés ni las habilidades para hacerlo. En tal sentido, hay programas que tienen dos vías, como se ha discutido antes, agregando un año para los residentes interesados en investigación $^{(4)}$. Esto implicaría un cambio de la estructura misma del residentado, lo cual es complejo pero no imposible. Así, por ejemplo, la Escuela de Medicina de la Universidad de Puerto Rico tiene un programa posdoctoral de maestría en investigación clínica y traslacional, que permite a los residentes hacer una transición a ser investigadores médicos o "physician scientist ${ }^{\text {,(13) }}$. Actualmente, están abriendo la opción de la vía de investigación para los residentes de medicina interna interesados, extendiendo la residencia a cuatro años y así poder cumplir con los requerimientos clínicos y de investigación. Esto les permite terminar el residentado y la maestría durante ese período. Esta es una alternativa muy interesante para nuestro país, dado que según la nueva Ley Universitaria se necesita tener el grado de maestro para poder ser profesor universitario. Si bien, hay una excepción para los médicos con especialización para hacer carrera universitaria, se requiere del grado de maestro para ser profesor asociado, y el grado de doctor para ser profesor principal.

\section{Comentario final y sugerencias}

En base a lo arriba planteado, me gustaría discutir la relevancia y utilidad de que los residentes realicen un proyecto de investigación de la especialidad sin tener que ejecutarlo, según lo indica el nuevo reglamento del residentado. Al igual que otros mencionados ${ }^{(2)}$, dudo que esta normativa realmente contribuya en la capacitación médica y científica de los residentes. La norma es relevante pues ayudará a incrementar el número de residentes que se titulan, pues esto era un problema con el reglamento anterior. Por otro lado, en el proceso de redacción del proyecto, los residentes adquirían algunas habilidades de investigación incluyendo la revisión detallada de la literatura, la elaboración de la pregunta de investigación y el diseño en si del estudio. Pero, me parece que a la larga este ejercicio no contribuirá en mucho a su formación dado que es un producto estéril, que no llevará a ningún puerto: no se 
residency. Can Med Educ J. 2014;5(1):e58-61.

6. Ticse R, Pamo O, Samalvides F, Quispe T. [Associated factors with the completion of research project required to obtain a specialist title in a Peruvian University]. Rev Peru Med Exp Salud Publica. 2014;31(1):48-55.

7.Concha M, Miranda J C, Vergara G, Ibarra $H$. [Commitment and development of competences in clinical research in residency training programs in Chile]. Rev Med Chil. 2012;140(3):326-333.

8. Clarke PM. Overcoming obstacles to research in residency. JAMA. 2013;309(11):1109-1110.

9.Saldaña-Gastulo JJ, Quezada-Osoria CC, Peña-Oscuvilca A, Mayta-Tristán P. [High frequency of plagiarism in medical thesis from a Peruvian public university]. Rev Peru Med Exp Salud Publica. 2010;27(1):63-67.

10. Moreno-Loaiza O, Mamani-Quispe PV, Mayta-Tristán P. [Online buying and selling of thesis: an ethical problem to control]. Rev Peru Med Exp Salud Publica. 2013;30(2):352354.

11. Carnero AM, Mayta-Tristan P, Konda KA, Mezones-
Holguin E, Bernabe-Ortiz A, Alvarado GF, Canelo-Aybar C, Maguiña JL, Segura ER, Quispe AM, Smith ES, Bayer AM, Lescano AG. Plagiarism, Cheating and Research Integrity: Case Studies from a Masters Program in Peru. Sci Eng Ethics. 2017 ;23(4):1183-1197.

12.Himelhoch S, Edwards S, Ehrenreich M, Luber MP. Teaching Lifelong Research Skills in Residency: Implementation and Outcome of a Systematic Review and Meta-Analysis Course. J Grad Med Educ. 2015;7(3):445-450.

13. Hernandez-Suarez D, San Martin MT, Colon-Marquez JA, Jimenez-Velazquez IZ, Lopez-Candales A. Continued Need for Clinical Research in Medical Residency: From Physician to Clinical-Translational Researcher. P R Health Sci J. 2017;36(1):44-46.

14. Valle R, Perales A. [Health research in Peru]. Rev Peru Med Exp Salud Publica. 2016;33(4):833-834.

15. Pamo Reyna OG, Olascoaga Mesía AC. [New rules on the qualification of medical residency in Peru: problems and perspectives]. Rev Peru Med Exp Salud Publica. 2016;33(4):832-833.

Citar como: Ochoa-Woodell TJ. ¿Es necesario exigir investigación en el Residentado Médico? Diagnóstico (Lima). 2019;58(3):138-142.

DOI: 10.33734/diagnostico.v58i3.62

Correspondencia: Theresa J. Ochoa-Woodell Correo electrónico: Theresa.Ochoa@upch.pe

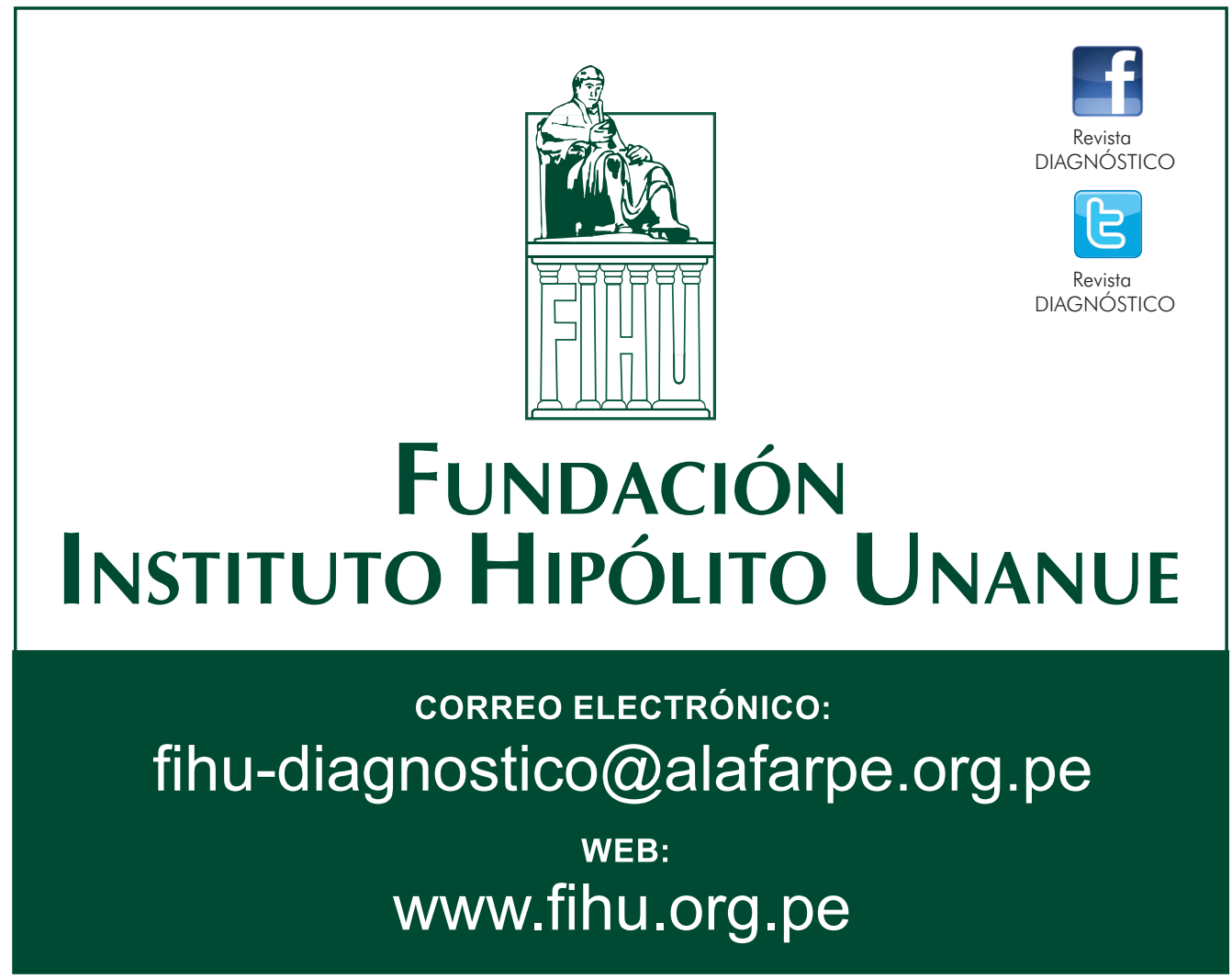

